\title{
A biomecânica e a produção do conhecimento em fisioterapia: levantamento baseado nos anais do congresso brasileiro de biomecânica
}

\author{
The biomechanics and the production of knowledge in physiotherapy: survey based on \\ the proceedings of Brazilian congress of biomechanics \\ Paula Hentschel Lobo da Costa', Roberta de Fátima Carreira Moreira², Fabiana Almeida Foltran², Luiz \\ Fernando Approbato Selistre ${ }^{2}$, Kleber Luis Silva Santos ${ }^{2}$, Kelli Cristina de Castro², Natália Targas Lima ${ }^{3}$, \\ Teresinha das Graças Coletta ${ }^{4}$
}

\begin{abstract}
RESUMO I O aumento gradual da participação da comunidade acadêmica da Fisioterapia nas edições do Congresso Brasileiro de Biomecânica (CBB) é notório. Os Anais do CBB passaram a ser importantes veículos para a divulgação científica em Fisioterapia no Brasil; porém, a caracterização dessa produção ainda não foi feita. O objetivo do presente estudo foi realizar um levantamento bibliográfico dos estudos em Fisioterapia publicados nas edições dos Anais do CBB, desde a primeira edição em 1992 (Anais do IV CBB) até a edição publicada em 2009 (Anais do XIII CBB), a fim de identificar quais especialidades da Fisioterapia têm aplicado o conhecimento em Biomecânica no contexto clínico e/ou científico, além de caracterizar o tipo de pesquisa que se tem desenvolvido. Seis revisores independentes levantaram os estudos pertencentes à área da Fisioterapia e coletaram informações de maneira padronizada através de questionários. Os resultados evidenciaram um grande crescimento da participação das diferentes áreas da Fisioterapia ao longo das dez edições do CBB. Embora os dados sejam positivos em relação à ampliação das pesquisas em Biomecânica, verificou-se uma carência da utilização dos recursos biomecânicos para avaliar efeitos de intervenções em pacientes. Dessa forma, recomenda-se que mais estudos sejam conduzidos em contextos clínicos e com acompanhamento longitudinal, de modo a ampliar a aplicação prática das ferramentas biomecânicas no campo da intervenção, bem como aperfeiçoar a avaliação em Fisioterapia. Descritores I biomecânica; fisioterapia; pesquisa.
\end{abstract}

\begin{abstract}
I The increasing presence of the Physical Therapy (PT) community in the Brazilian Congress of Biomechanics (CBB) is well known. The Proceedings of the CBB Congresses have turned to important vehicles to scientific publications in Physical Therapy, but the characteristics of these studies have not been focus of analysis. Thus, the purpose of this study was to identify the contribution of PT to the studies published in the Proceedings of the CBB Congresses, from the first edition in 1992 (Proceedings of the IV CBB) to the 2009 edition (Proceedings of the XIII CBB), in order to verify which subareas of expertise in PT are applying the biomechanical knowledge on clinical and/or scientific context and the kind of research being developed. Six independent reviewers collected data related to the PT production according to standardized questionnaires. The results evidenced that the area of PT has increased its publications along the ten CBB Proceedings. Despite positive findings, we noticed a lack of biomechanical studies aimed at investigating interventions in patients. Thus, we can suggest that clinical studies with a longitudinal design could be developed in order to improve the application of biomechanical tools in therapeutic settings and to contribute to evaluation procedures in PT.
\end{abstract}

Keywords I biomechanics; physical therapy specialty; research.

\footnotetext{
Estudo realizado na Universidade Federal de São Carlos (UFSCar) - São Carlos (SP), Brasil.

'Doutora em Biodinâmica do Movimento Humano, Departamento de Educação Física e Motricidade Humana da UFSCar - São Carlos (SP), Brasil. ${ }^{2}$ Programa de Pós-Graduação em Fisioterapia da UFSCar - São Carlos (SP), Brasil.

${ }^{3}$ Departamento de Educação Física e Motricidade Humana da UFSCar - São Carlos (SP), Brasil.

${ }^{4}$ Mestre em Engenharia de Produção, Escola de Engenharia de São Carlos da Universidade de São Paulo (USP) - São Carlos (SP), Brasil.
} 


\section{INTRODUÇÃO}

Todo movimento é um fenômeno mecânico e sempre que uma força atua sobre o corpo humano, direta ou indiretamente, princípios biomecânicos estão envolvidos. O estudo de forças é fundamental para a compreensão de situações estáticas e dinâmicas do movimento humano, seja ele normal ou patológico ${ }^{1}$. $\mathrm{O}$ estudo de forças que atuam sobre ou no corpo humano é feito pela Biomecânica.

Biomecânica pode ser considerada uma parte inerente à Fisioterapia ${ }^{2}$, pois, ao lidar com disfunções do movimento, a Fisioterapia precisa da descrição desta, feita pela cinemática. Uma cinemática anormal precisa ser explicada pelas forças atuantes sobre o corpo, geradoras do movimento. Para isso, princípios da cinética precisam ser considerados.

A Biomecânica foi considerada como uma área que, em 1984, ainda estava restrita à pesquisa em Fisioterapia nos Estados Unidos ${ }^{1}$. No Brasil, não é possível inferir sobre o papel exato da Biomecânica na formação inicial de fisioterapeutas, pois não foram encontrados estudos na literatura sobre esse tema. Ainda em 1984, Devine ${ }^{3}$ afirmou que currículos em Fisioterapia deveriam considerar o desenvolvimento de competências biomecânicas para atuação em áreas de prevenção de disfunções do movimento, avaliação e tratamento de pacientes, a fim de se superar o enfoque estritamente de pesquisa.

$\mathrm{O}$ investimento em pesquisa na Fisioterapia tem crescido rapidamente nos últimos anos e um importante marco para esse desenvolvimento foi a criação, em 2005, da Associação Brasileira de Pesquisa e Pós-Graduação em Fisioterapia ${ }^{4}$. Entretanto, antes disso já era notório o aumento gradual da participação da comunidade acadêmica da Fisioterapia nas edições do Congresso Brasileiro de Biomecânica (CBB), o que refletia o interesse dos pesquisadores pela área. Verificou-se um crescimento gradual tanto do número absoluto de trabalhos, quanto da participação relativa da Fisioterapia nos Anais do $\mathrm{CBB}$, desde a primeira edição dos Anais em 1992, até a publicada em 2009, na qual 56,5\% dos estudos originaram-se em Departamentos de Fisioterapia ${ }^{5}$.

Esse crescimento do interesse da Fisioterapia pela pesquisa em Biomecânica teve como consequência a necessidade de espaço para a divulgação de novos conhecimentos e para discussões sobre os avanços científicos e técnicos.

Os Anais do CBB passaram a ser importantes veículos para a divulgação científica em Fisioterapia no Brasil; porém, a caracterização dessa produção ainda não foi feita. As tendências de publicações nas edições dos anais do $\mathrm{CBB}$ foram analisadas previamente por Mochizuki et al. ${ }^{6}$, Cotta e Mochizuki ${ }^{7}$; porém, esses estudos não fizeram referência à contribuição relativa de áreas específicas do conhecimento.

Os Anais entre 1992 e 2005 foram avaliados por Cotta e Mochizuki ${ }^{7}$. Os autores focaram a discussão na distribuição dos estudos quanto à metodologia biomecânica utilizada e identificaram uma redução percentual dos estudos sobre instrumentação biomecânica e de revisão bibliográfica, a partir da edição de 2001. Por outro lado, observaram um crescimento dos estudos experimentais, identificando a dinamometria como a metodologia mais utilizada. Não foram feitas considerações sobre quais áreas do conhecimento estavam contribuindo para essa produção.

Também Mochizuki et al. ${ }^{6}$ avaliaram a produção dos anais do CBB de 1992 a 2003, usando um modelo matemático para descrever o ritmo de crescimento da produção e identificaram a região Sudeste como a origem da maior parte das publicações brasileiras.

A qualidade da pesquisa em Fisioterapia publicada em congressos foi analisada por Coury ${ }^{8}$. A tendência ao longo de 20 anos dos estudos destinados à intervenção ergonômica foi avaliada sistematicamente a partir de publicações de 7 Congressos Latino-Americanos de Ergonomia e 12 Congressos Brasileiros de Ergonomia. A autora identificou que apenas uma pequena porcentagem dos estudos abordava medidas de intervenção preventivas e uma porcentagem ainda menor destes apresentava conclusões convincentes.

Com vistas a melhor identificar quais especialidades da Fisioterapia têm aplicado o conhecimento em Biomecânica no contexto clínico e/ou científico, e caracterizar o tipo de pesquisa que se tem feito, o presente estudo apresenta como objetivo realizar um levantamento bibliográfico dos estudos em Fisioterapia publicados nos Anais do $\mathrm{CBB}$, desde a primeira edição em 1992 até a publicada em 2009.

O levantamento das características das pesquisas em Fisioterapia publicadas nos anais do CBB poderá contribuir para identificar as subáreas envolvidas com a Biomecânica e a forma como esta tem contribuído para a atuação profissional na área.

\section{MATERIAIS E MÉTODOS}

\section{Estratégia de busca}

Foi realizada pesquisa nas edições dos anais do CBB publicadas desde a primeira edição até 2009, o que 
compreendeu as edições de $1992^{9}, 1993^{10}, 1995^{11}$, $1997^{12}, 1999^{13}, 2001^{14}, 2003^{15}, 2005^{16}, 2007^{17}$ e $2009^{18}$.

Para a identificação do estudo como pertencente à área da Fisioterapia, foram inicialmente verificados o título do estudo e a filiação dos autores. Em caso de ausência desses dados, foi consultado o currículo Lattes. Durante essa consulta, considerou-se que se ao menos um dos autores pertencesse a um departamento de Fisioterapia, o estudo seria considerado para análise.

Inicialmente, seis revisores independentes trabalharam coletando dados de todos os estudos que compunham os anais. Todos os estudos foram classificados de acordo com a área do conhecimento a que pertenciam. Os revisores utilizaram dois formulários padronizados que auxiliaram a coleta de dados gerais e específicos sobre os trabalhos publicados. Eventuais dúvidas e discordâncias durante o processo foram solucionadas por meio de consenso.

A seguir, todos os estudos relacionados à Fisioterapia foram selecionados e tiveram seus resumos analisados para identificar o assunto abordado e a subárea específica a que pertenciam.

\section{Critérios de inclusão}

\section{Tipos de participantes}

Foram selecionados apenas estudos que relataram resultados referentes a seres humanos, independentemente do gênero, faixa etária e condição de saúde.

\section{Tipo de resultados relatados}

Foram selecionados os estudos que utilizaram instrumentação específica para avaliação de variáveis biomecânicas em seres humanos.

\section{Extração dos dados}

Todos os revisores trabalharam de forma independente, utilizando dois formulários padronizados para a extração dos dados. Inicialmente, através do formulário 1 , todos os estudos apresentados nas 10 edições do $\mathrm{CBB}$ foram classificados quanto a: 1) edição do $\mathrm{CBB}$ a que pertencia; 2) área do conhecimento; 3) instituição à qual, ou às quais, seus autores estavam filiados.

A seguir, por meio do formulário 2, os estudos relacionados à Fisioterapia foram analisados separadamente, sendo então classificados quanto a: 1) instituição de ensino; 2) subárea da Fisioterapia a que pertencia;
3) características da população do estudo, como o perfil dos sujeitos avaliados, número de sujeitos, média de idade e gênero; 4) desenho do estudo, classificado entre dois modelos: transversal e longitudinal; 5) variáveis biomecânicas avaliadas e 6) instrumentação utilizada.

Após a tabulação dos dados, foram criadas novas categorias para facilitar a análise dos resultados. Assim, as instituições de ensino foram agrupadas de acordo com a região geográfica a que pertenciam. Os sujeitos foram categorizados em acometidos e não acometidos de acordo com a presença ou ausência de afecções do sistema musculoesquelético. Em relação à idade, foram estabelecidas 4 categorias de acordo com a seguinte faixa etária: crianças (0 a 15 anos); adultos jovens (de 16 a 29 anos); adultos (30 a 59 anos) e idosos (idade superior a 60 anos). Quanto à instrumentação utilizada, foram usadas quatro categorias baseadas nas variáveis biomecânicas medidas ${ }^{19}$ : antropometria, cinemetria, eletromiografia, dinamometria e outros (incluindo metodologias de goniometria, eletrogoniometria, manovacuometria, testes clínicos, fleximetria e termometria).

Não foi realizada avaliação da qualidade metodológica dos estudos incluídos, pois havia uma grande variedade de modelos experimentais, o que inviabilizou a padronização dos estudos para uma avaliação objetiva e comparativa da qualidade.

Finalmente, foi realizada uma avaliação geral sobre a organização dos anais considerando: as áreas utilizadas para classificação dos trabalhos; a forma de apresentação dos estudos; a presença de sumário e de índice de autores e o meio de divulgação dos anais.

\section{Análise de dados}

Os resultados foram analisados de forma descritiva levando em consideração a distribuição de frequência dos resultados obtidos.

\section{RESULTADOS E DISCUSSÃO}

\section{Participação da Fisioterapia}

Ao longo das edições do CBB, a Fisioterapia participou de maneira cada vez mais significativa quanto ao total de publicações. Observa-se que a partir do ano de 2001 houve incremento dessa participação. A contribuição da Fisioterapia, de forma geral, passou de 2,4\% do total de estudos em 1992 para 56,5\% em 2009 (Tabela 1). 
Além do aumento na quantidade de estudos, diferentes áreas da Fisioterapia foram se apresentando nas edições do $\mathrm{CBB}$, demonstrando maior vínculo com a instrumentação biomecânica.

A Ortopedia destacou-se em número de publicações no $\mathrm{CBB}$ desde as primeiras edições, seguida pela Ergonomia (participação regular desde 1997) e Neurologia (desde 1999). Os estudos em metodologia de pesquisa em Fisioterapia e a participação de outras áreas como a Fisioterapia respiratória, reumatológica, cardiológica e ginecológica (agrupadas na categoria denominada 'Outras') já é fato desde a edição de 1999 (Figura 1).

Provavelmente o predomínio da Ortopedia se deva à ampla utilização de conceitos e princípios biomecânicos na área para fins clínicos e de ensino, dando suporte à pesquisa e ao desenvolvimento de tecnologia como os dinamômetros isocinéticos.

Tabela 1. Distribuição de estudos da Fisioterapia em porcentagem (\%) do total de publicações e em valores absolutos ao longo das edições dos Anais do Congresso Brasileiro de Biomecânica

$\begin{array}{lcc}\begin{array}{l}\text { Edição dos Anais } \\ \text { do CBB }\end{array} & \begin{array}{c}\text { Porcentagem do } \\ \text { total de estudos }\end{array} & \begin{array}{c}\text { Total absoluto de } \\ \text { estudos }\end{array} \\ 1992 & 2,4 & 1 \\ 1993 & 0 & 0 \\ 1995 & 6,1 & 4 \\ 1997 & 12,3 & 10 \\ 1999 & 13,9 & 14 \\ 2001 & 25,0 & 26 \\ 2003 & 29,8 & 57 \\ 2005 & 46,3 & 137 \\ 2007 & 32,9 & 104 \\ 2009 & 56,5 & 160\end{array}$

CBB: Congresso Brasileiro de Biomecânica

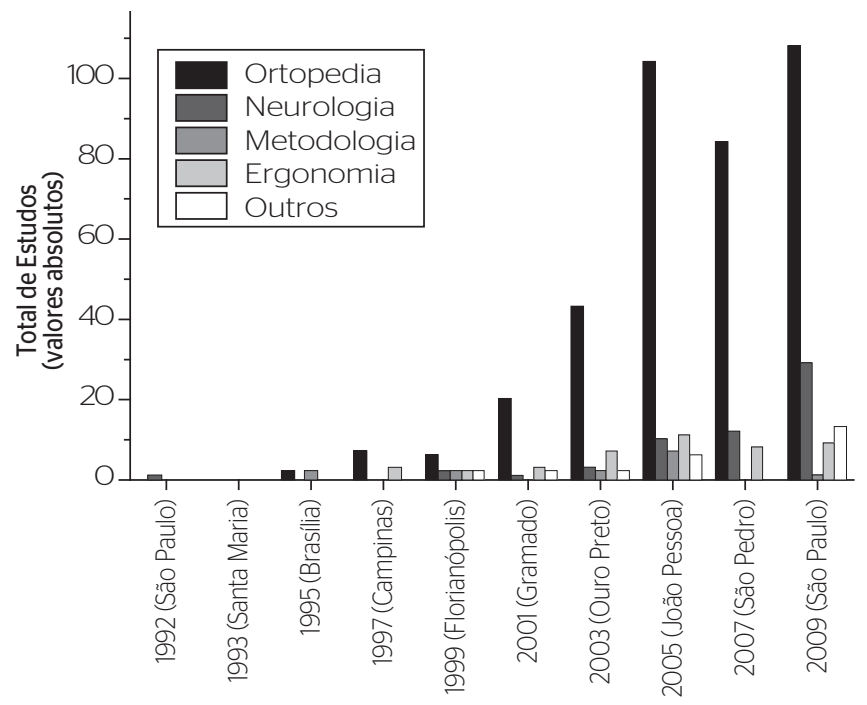

Edições dos Anais do CBB

CBB: Congresso Brasileiro de Biomecânica

Figura 1. Distribuição de estudos (em valores absolutos) por subárea da Fisioterapia nas edições dos Anais do Congresso Brasileiro de Biomecânica

\section{Características dos sujeitos de pesquisa}

Quanto à caracterização dos sujeitos de pesquisa, observou-se que os sujeitos sem acometimentos ou lesões (não acometidos) foram mais pesquisados que aqueles com algum tipo de distúrbio neuromusculoesquelético (Tabela 2). Esse tipo de população passou a predominar na edição do ano de 2003, talvez pela facilidade de recrutamento. Quanto à faixa etária dos sujeitos de pesquisa, verificou-se que, de um modo geral, todas as faixas etárias estiveram presentes nos estudos, contudo houve predomínio da faixa etária composta por adultos jovens (Tabela 2). Além disso, vale ressaltar que na edição de 2009 houve grande quantidade de trabalhos (34\%) sem informação quanto à idade, o que dificultou a classificação em populações específicas quanto à faixa etária. A análise das amostras demonstrou que um grande número de estudos utilizou estudantes universitários para a obtenção de dados. O fácil acesso a essa população, sua relativa disponibilidade para participar de protocolos experimentais são fatores que possivelmente contribuíram para tal fato.

Esses resultados demonstram que tem havido pouca aplicabilidade das metodologias biomecânicas no contexto clínico. Tal fato pode estar relacionado à dificuldade de acesso aos equipamentos, à ausência de espaços apropriados para sua utilização nos setores de atendimento, bem como a problemas de acesso relacionados ao transporte e deslocamento de pacientes para laboratórios de universidades. Assim, a afirmação de Devine ${ }^{3}$ continua atual, no sentido de que permanece no Brasil a necessidade de se superar o enfoque estritamente de pesquisa da Biomecânica em Fisioterapia.

\section{Instrumentação utilizada}

A instrumentação utilizada nos estudos foi classificada de acordo com a variável biomecânica mensurada. Observou-se que a dinamometria prevaleceu como metodologia de avaliação a partir do ano de 1999 e que a cinemetria passou a ser utilizada de forma mais regular na pesquisa em Fisioterapia na edição de 1997. Porém, a partir de 2005 observou-se um expressivo crescimento do emprego da cinemetria (Figura 2). Neste estudo, a cinemetria incluiu as metodologias de radiografia, ultrassonografia e ressonância magnética, sempre que as informações foram quantificadas, do contrário, quando esses métodos eram empregados apenas para efeito de diagnóstico, os estudos não foram incluídos na análise. A dinamometria incluiu as metodologias de 
Tabela 2. Características dos sujeitos quanto à presença de distúrbios neuromusculoesqueléticos e faixa etária em valores absolutos de estudos nas edições dos Anais do Congresso Brasileiro de Biomecânica

\begin{tabular}{|c|c|c|c|c|c|c|c|}
\hline \multirow{2}{*}{$\begin{array}{l}\text { Edição } \\
\text { dos anais }\end{array}$} & \multicolumn{2}{|c|}{ Característica } & \multicolumn{5}{|c|}{ Faixa etária } \\
\hline & acometido & não acometido & idoso & adulto & adulto jovem & criança & sem informação de idade \\
\hline 1992 & 1 & & & & & 1 & \\
\hline \multicolumn{8}{|l|}{1993} \\
\hline 1995 & 1 & 1 & & & 1 & & 1 \\
\hline 1997 & 2 & 8 & & 1 & 7 & 1 & 1 \\
\hline 1999 & 8 & 5 & 2 & & 4 & 2 & 6 \\
\hline 2001 & 20 & 7 & 1 & & 23 & 1 & 2 \\
\hline 2003 & 11 & 44 & 4 & 7 & 37 & 5 & 2 \\
\hline 2005 & 33 & 43 & 7 & 33 & 59 & 17 & 16 \\
\hline 2007 & 39 & 45 & 7 & 25 & 40 & 10 & 16 \\
\hline 2009 & 71 & 91 & 26 & 24 & 55 & 12 & 55 \\
\hline
\end{tabular}

dinamometria isocinética e baropodometria dinâmica. A categoria 'Outros' incluiu: impressão plantar, goniometria, eletrogoniometria, termometria, monofilamentos e manovacuômetro.

\section{Distribuição geográfica dos núcleos de pesquisa}

A classificação baseada na localização geográfica das instituições (Figura 3) mostrou que em 1992 e 1993 não houve participação da fisioterapia nacional nas produções do CBB, apenas uma participação internacional em 1992. Porém, a partir de 1999 a produção em Fisioterapia das regiões sudeste e sul começou a se fazer presente nos Anais do $\mathrm{CBB}$, com crescimento rápido ao longo das edições, principalmente no sudeste. A região nordeste passa a publicar nos anais no ano de 1997 . No ano de 2005, observou-se a presença de produções científicas das regiões norte e centro-oeste, além da região nordeste ter atingido o auge de sua produção. Nesse ano o congresso foi realizado em João Pessoa (PB), o que pode ter contribuído para esse fato. Desde então, as regiões norte, nordeste e centro-oeste mantém com regularidade sua produção nas edições do $\mathrm{CBB}$. Esse fato foi mais bem avaliado, pois havia o indicativo de que houvesse uma clara relação entre o local sede do congresso e o aumento da publicação dos grupos locais e regiões. No entanto, esse fato não se comprovou nas outras edições dos Anais do CBB.

Há uma parceria internacional na primeira edição em 1992, mas apenas em 1999 houve novamente esse tipo de estudo, mantendo uma regularidade de contribuição a partir de 2003. Essas parcerias internacionais refletem o interesse na formação de recursos humanos em Biomecânica e a busca pelo domínio de novas metodologias de pesquisa. Tais parcerias são importantes e devem ser incentivadas, de modo a aprimorar as

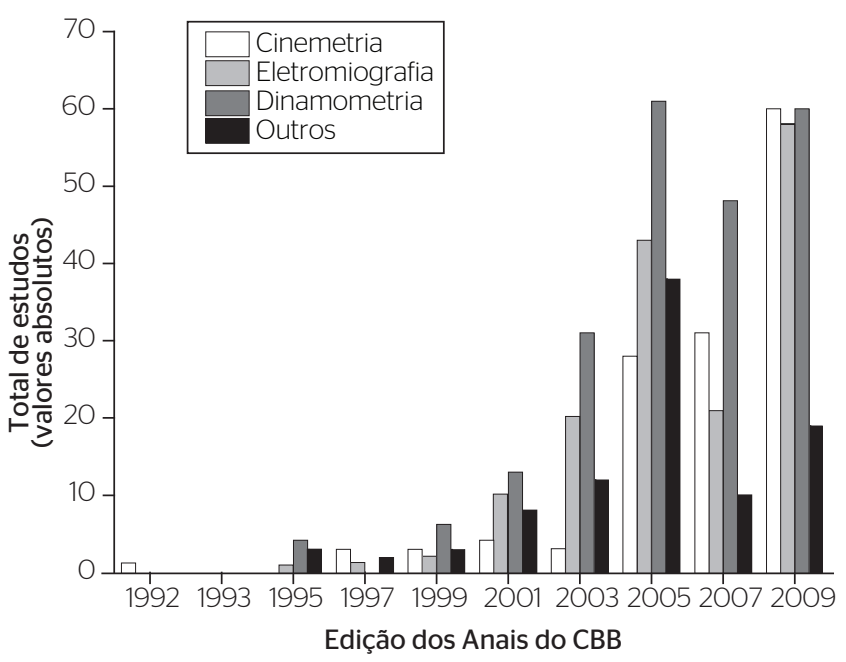

CBB: Congresso Brasileiro de Biomecânica

Figura 2. Instrumentação biomecânica utilizada em totais absolutos de estudos

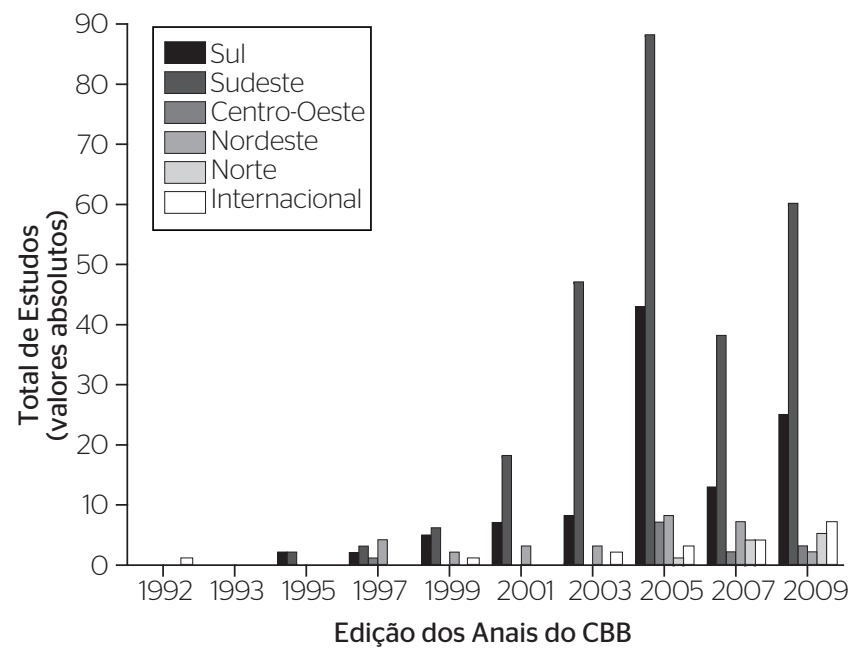

CBB: Congresso Brasileiro de Biomecânica

Figura 3. Distribuição dos estudos (valores absolutos) por região do Brasil e presença de parcerias internacionais

pesquisas nacionais e fortalecer as cooperações internacionais para pesquisa na área de Biomecânica aplicada à Fisioterapia. 


\section{Desenho dos estudos}

A análise do desenho dos estudos demonstrou que em 1992 o único estudo da Fisioterapia era uma parceria internacional e tinha caráter longitudinal. Em 1995, foram identificados um estudo longitudinal e um transversal. A partir de 2001, houve grande predomínio de desenhos transversais de estudos, que passaram a corresponder a $92,89,74,76$ e $81 \%$ nos anos de 2001, 2003, 2005, 2007 e 2009, respectivamente. Observou-se que a maioria dos estudos envolveu questões específicas, desenvolvidas em contextos com pouca possibilidade de generalização dos resultados.

\section{Considerações sobre a apresentação dos Anais}

O presente estudo não se propõe à avaliação da qualidade metodológica das publicações dos Anais do CBB e nem a sintetizar evidências sobre um aspecto de pesquisa específico. O objetivo foi apresentar um panorama da pesquisa em Biomecânica na Fisioterapia, indicando os caminhos que têm sido traçados de acordo com os documentos avaliados.

Um aspecto muito importante nessa análise foi o levantamento da normatização dos anais. Observou-se que há, de maneira geral, uma carência de normatização, o que se reflete em publicações (tanto impressas, quanto na forma digital) não padronizadas. A classificação dos estudos em áreas da Biomecânica na composição dos Anais precisa ser mais bem definida, pois há estudos metodologicamente semelhantes que se inserem em áreas diferentes no mesmo livro de Anais. O mesmo ocorre na comparação de Anais de edições diferentes. Ainda, um mesmo estudo pode ser incluído em mais de um assunto ou subárea, criando uma classificação por vezes dúbia. A fim de superar essa limitação, nas próximas edições poderia ser utilizado um índice comum para áreas e subáreas, por exemplo, definido a partir de um vocabulário controlado.

Alguns anais estão organizados pela forma de apresentação, outros pelo tipo de publicação e ainda há a organização com base na ordem de inscrição dos estudos no CBB. Esse fato dificultou a classificação dos trabalhos, o uso de instrumentos de busca e, certamente, o acesso aos documentos. Todas as dificuldades relativas à falta de normatização dos anais podem ter afetado os resultados do presente estudo, representando um fator limitante de nossas conclusões.
Anais de eventos elaborados apenas na forma digital têm inúmeras vantagens; porém, caso não sejam construídos a partir de plataformas automáticas, com padronização de templates para os diferentes formatos de publicação (resumos, artigos, completos), ou formas de apresentação (tema oral, pôster, conferência) que utilizem robôs de busca (por palavras-chave, sobrenome do autor, entre outros), tornam a recuperação do documento uma tarefa difícil. Naturalmente, a disseminação do conhecimento depende em grande parte da facilidade de acesso aos documentos por parte dos interessados.

Finalmente, o baixo grau de citação dos estudos, mencionado por Mochizuki et al. ${ }^{6}$ pode ser decorrente da dificuldade de acesso aos documentos devido à deficiente normatização dos anais e não necessariamente pela pouca relevância ou novidade do conhecimento veiculado.

\section{CONCLUSÃO}

Houve um crescimento relevante da participação das diferentes áreas da Fisioterapia ao longo das dez edições do CBB incluídas na avaliação proposta por este estudo. Esse crescimento pode refletir: 1) o aumento do interesse pelo uso da Biomecânica na pesquisa em Fisioterapia; 2) um maior domínio dos recursos disponíveis, a partir de parcerias que favorecem a troca de informação e o compartilhamento da instrumentação; 3) um maior acesso às novas tecnologias que permitem aliar informações quantitativas aos dados qualitativos relacionados às avaliações clínicas do movimento humano.

Embora esses resultados sejam positivos em relação à ampliação do uso das ferramentas da Biomecânica nas pesquisas desenvolvidas por departamentos de Fisioterapia, verificou-se uma carência da utilização dessas ferramentas para avaliação objetiva dos efeitos de intervenções fisioterapêuticas em indivíduos com disfunção.

A maioria dos estudos avaliados não foi realizada dentro de um contexto terapêutico. Tal fato foi evidenciado pelo grande número de estudos com desenho transversal e pelo predomínio da participação de indivíduos saudáveis. Assim, é possível reforçar a importância da aplicação prática das competências biomecânicas utilizadas na pesquisa durante a atuação terapêutica. Além disso, enfatiza-se a necessidade de desenvolvimento de ensaios clínicos futuros utilizando as ferramentas biomecânicas disponíveis para avaliação dos efeitos de intervenções clinicas. 
Dessa forma, recomenda-se que mais estudos sejam conduzidos em contextos clínicos e com acompanhamento longitudinal de modo a ampliar a aplicação prática das ferramentas biomecânicas no campo da intervenção, bem como aperfeiçoar a avaliação em Fisioterapia.

Ressalta-se a importância de adotar normatização padronizada de publicações visando facilitar a recuperação de dados e o acesso aos documentos publicados, independentemente da forma de divulgação, impressa ou digital.

\section{REFERÊNCIAS}

1. Leveau BF. Biomechanics: a summary of perspectives. Phys Ther. 1984;64(12):1812.

2. Smidt GL. Biomechanics and physical therapy: a perspective. Phys Ther. 1984;64(12):1807-8

3. Devine K. Competencies in Biomechanics for the physical therapist: suggestion for entry-level curricula. Phys Ther. 1984;64(12):1883-5.

4. Andrade AFD, Britto RR. Unindo forças seremos fortes! Associação Brasileira de Pesquisa e Pós-Graduação em Fisioterapia. Rev Bras Fisioter. 2008;12(6):5-6.

5. Moreira RFC, Selistre LFA, Foltran FA, Santos KL, Castro KC, Lima NT, Lobo da Costa, PH. Contribuição da Fisioterapia para a pesquisa em biomecânica no CBB: uma revisão sistemática. Anais do XIV Congresso Brasileiro de Biomecânica; 2011 Jun 9-11; Ribeirão Preto SP. São Paulo: Sociedade Brasileira de Biomecânica; 2011. p. 114.

6. Mochizuki L, Franciulli PM, Bigongiari, Araujo RC, Serrão JC, Amadio AC. Análise do impacto das publicações do Congresso Brasileiro de Biomecânica. Anais do XI Congresso Brasileiro de Biomecânica; 2005 Jun 18-22; João Pessoa PB, São Paulo: Sociedade Brasileira de Biomecânica; 2005.
7. Cotta RF, Mochizuki L. Análise das tendências de pesquisa em Biomecânica. XIII CBB, 2009. Anais do XIII Congresso Brasileiro de Biomecânica: 2009 Jul 29-01, São Paulo, SP, Brasil. São Paulo: Sociedade Brasileira de Biomecânica; 1999.

8. Coury HJCG. Time trends in ergonomic intervention research for improved musculoskeletal health and comfort in Latin America. Appl Ergon. 2005;36(2):249-52.

9. Anais do IV Congresso Nacional de Biomecânica; 1992 Dez 2-4; São Paulo, SP, Brasil. São Paulo: Sociedade Brasileira de Biomecânica; 1992.

10. Anais do V Congresso Brasileiro de Biomecânica; 1993 Dez 1-3; Santa Maria, RS, Brasil. São Paulo: Sociedade Brasileira de Biomecânica; 1993.

11. Anais do VI Congresso Brasileiro de Biomecânica; 1995 Mai 24-26; Brasília, DF, Brasil. São Paulo: Sociedade Brasileira de Biomecânica; 1995.

12. Anais do VII Congresso Brasileiro de Biomecânica; 1997 Mai 23-30; Campinas, SP, São Paulo: Sociedade Brasileira de Biomecânica; 1997.

13. Anais do VIII Congresso Brasileiro de Biomecânica; 1999 Mai 2629; Florianópolis, SC, Brasil. São Paulo: Sociedade Brasileira de Biomecânica; 1999

14. Anais do IX Congresso Brasileiro de Biomecânica; 2001 Jun 29-01; Gramado, SC, Brasil. São Paulo: Sociedade Brasileira de Biomecânica; 2001.

15. Anais do X Congresso Brasileiro de Biomecânica; 2003 Jun 3-6; Ouro Preto, SP, Brasil. São Paulo: Sociedade Brasileira de Biomecânica; 2003.

16. Anais do XI Congresso Brasileiro de Biomecânica; 2005 Jun 1822; João Pessoa, PB, Brasil. São Paulo: Sociedade Brasileira de Biomecânica; 2005.

17. Anais do XII Congresso Brasileiro de Biomecânica; 2007 Mai 30-02; São Pedro, SP, Brasil. São Paulo: Sociedade Brasileira de Biomecânica; 2007.

18. Anais do XIII Congresso Brasileiro de Biomecânica; 2009 Jul 28-01; São Paulo, SP, Brasil. São Paulo: Sociedade Brasileira de Biomecânica; 2009

19. Amadio AC, Duarte M (Eds). Fundamentos biomecânicos para a análise do movimento humano. São Paulo: EEFEUSP; 1996. 